Landslides (2021) 18:1349

DOI 10.1007/s10346-020-01600-6

Published online: 17 December 2020

(c) Springer-Verlag GmbH Germany

part of Springer Nature 2020

\section{Francesco Bregoli · Vicente Medina · Allen Bateman \\ Correction to: The energy transfer from granular landslides to water bodies explained by a data-driven, physics-based numerical model}

\section{Correction to: Landslides.}

https://doi.org/10.1007/s10346-020-01568-3

The published version of this article contains error. The author noticed that there are many mistakes in the formatting and layout the paper. These include author affiliation, equations, figure resolution, open access license, Table 1 layout and some of the references.

The original article has been corrected.

The online version of the original article can be found at https://doi.org/10.1007/s10346020-01568-3

\author{
F. Bregoli $(-)$ A. Bateman \\ Sediment Transport Research Group (GITS), Department of Hydraulic, Marine and \\ Environment Engineering, \\ Universitat Politècnica de Catalunya, \\ UPC Campus Nord, Building D1, C/ Jordi Girona 1-3, 08034, Barcelona, Spain \\ Email: f.bregoli@science.ru.nl
}

Present address:

Environmental Science Department, Institute for Water and Wetland Research, Radboud University, Heyendaalseweg 135, 6525 AJ, Nijmegen, the Netherlands

E-mail: f.bregoli@science.ru.nl

A. Bateman

Email: allen.bateman@upc.edu

V. Medina

Thermal Engines Reseach Group (CREMIT), Department of Thermal Engines, Universitat Politècnica de Catalunya,

UPC Campus Sud, Avinguda Diagonal, 647, 08028, Barcelona, Spain

Email: vicente.de.medina@upc.edu 